\title{
Integrated Health Counseling Utilizing SMART Technology
}

\author{
Thomas W Miller ${ }^{1 *}$, Deborah Burton ${ }^{2}$ and Christina Busse ${ }^{3}$ \\ ${ }^{1}$ Department of Psychiatry, College of Medicine, University of Kentucky, USA \\ ${ }^{2}$ Manager, Telehealth Services, Kentucky One Health, Kentucky, USA \\ ${ }^{3}$ Community Outreach and Transitions of Care, Catholic Health Network, Kentucky One Health, Kentucky, USA
}

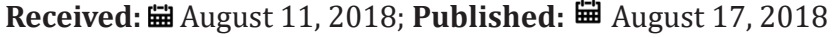

*Corresponding author: Thomas W Miller, Department of Psychiatry, College of Medicine, University of Kentucky, USA

\begin{abstract}
Examined is the use of a current model of interdisciplinary health care and the use of smart wearable technology for self-monitoring behavior change with a patient receiving prescribed outpatient counseling involving a registered dietician, health counselor and clinical psychologist. An introduction to SMART technology along with a relevant literature review is offered. It is hypothesized that the use of a wearable self-monitoring device may be beneficial in achieving behavioral compliance in a patient receiving mental health counseling. An exploratory case study research design is employed to examine this hypothesis. Results suggest that wearable SMART technology is an emerging adjunct effective intervention for improved health and well-being as measured by health and wellness markers utilizing a wearable SMART technology like the FitBit. Current limitations along with future directions are discussed. Mental health counselors may benefit from the utilization of wearable SMART technology as an adjunct when treating patients who experience anxiety related to their medical conditions.
\end{abstract}

Abbreviations: SMART Technology; E-Health; Dialectic Behavior Therapy

\section{Introduction}

SMART technology has opened a new frontier in both physical and mental health support and monitoring during counseling. SMART stands for Self-Monitoring Analysis and Reporting Technology. Mobile devices such as smartphones, and tablets are giving the clients, health care providers, and researcher's new ways to monitor progress, and increase understanding of health and wellbeing. E-health is a relatively recent term for healthcare practice utilizing technology-based assessment and treatment strategies that have the potential to deliver benefit to a broad array of individuals who experience anxiety related to their medical conditions [13]. SMART technology is a form of synchronous or asynchronous healthcare delivery that may serve as an adjunct to standardized forms of clinical treatment intervention. Appropriately trained and licensed professionals are entering the technology driven twenty first century health care arena. Professional organizations have recognized that with the use of current technology in the provision of health related counseling care and treatment, further professional standards and guidelines are necessary.

The American Counseling Association [4] Principal \#6 in addressing this domain noting that: Counselors actively attempt to understand the evolving nature of the profession with regard to distance counseling, technology, and social media and how such resources may be used to better serve their clients. Counselors strive to become knowledgeable about these resources. Counselors understand the additional concerns related to the use of distance counseling, technology, and social media and make every attempt to protect confidentiality and meet any legal and ethical requirements for the use of such resources. (Principle 6: Telehealth, Distance Counseling and the Use of Social Media)

Furthermore, within the realm of knowledge, skills, competencies and legal issues, "Counselors who engage in the use of distance counseling, technology, and/or social media develop knowledge and skills regarding related technical, ethical, and legal considerations [4]. The American Mental Health Counselors Association guides professional members that when members use Telehealth, Distance Counseling and the Use of Social Media "Counselors only provide distance counseling when they have had training, experience, and supervision to do so." [5].

Already addressed by the National Board for Certified Counselors is their NBCC's code of ethics which provides the ethical standard of practice that NCCs shall provide only those services for which they are qualified by education and experience. NCCs shall also consider their qualifications to offer such service via distance means. [6]. Similarly, the American Medical Association, Academy of Nutrition and Dietetics, American Psychological Association, and the National Association of Social Workers citations strongly indicate that education and training are part of gaining competence to utilize e-health skills and services. 
Contemporary models of medically supervised healthcare include an integrated clinical delivery model that emphasize multidisciplinary teams treating clients. Mental health counselors are recognized as bringing specialized skills to the multidisciplinary integrated health care team. More specifically, mental health counselors are emerging as a primary provider in the treatment of patients with both physical and mental health issues. In treating the whole person, managed care often requires an integrated team approach that addresses both physical and mental health components in health care delivery. Because of managed care, such a model of care requires a limited number of appointments that demonstrate measurable improvements in the current managed care environment. Wearable SMART technology provides a potential adjunct for use in assessing the therapeutic progress of some behavioral activities of appropriate clients.

\section{Literature Review}

Recent clinical guidelines and research [1,7-9] has identified the value and benefits of wearable technology for various populations raising the question as to whether there may be beneficial use with individuals in treatment for health related conditions. Wearable SMART technology employs digital technologies to collect health data from individuals in one location, such as a client's home, and electronically transmits the information to healthcare providers in a different location for assessment, monitoring and compliance [10-12].

SMART technology use and e-health [13] allows health care professionals to evaluate, diagnose and treat patients in remote locations using telecommunications technology. Smart wearable technologies are viewed as noninvasive digital technologies. Chan, Estève et al. [10] examined the use of SMART wearable systems in a broad area of health and wellness interventions significant for both physical and mental health. The results of their research concluded that advances in SMART technologies and subsequent SMART devices, ranging from sensors and actuators to multimedia devices, are beneficial in assessing change and monitoring patient compliance.

There is a patient population, that includes those individuals enrolled in health-related counseling and therapy, where the intervention is consistent with the cited definition of telemedicine and remote patient monitoring was employed using noninvasive digital technology for physical and psychological conditions. Such interventions have used devices including smartphones and PDA devices. A personal digital assistant (PDA) is a handheld device that combines computing, telephone/fax, Internet and networking features. A typical PDA can function as a cellular phone, fax sender, web browser and personal organizer. PDAs may also be referred to as a palmtop, hand-held computer or pocket computer. There is associated software used to transmit patient data to the patient's health care professional, including multiple health care providers and researchers. Such wearable devices worn or placed on one's body record a number of bio-physiological markers including such things as respiratory rate sensors, sleep and waking patterns of activity and blood pressure. There are also biosensor devices for recording data from biological or chemical reactions such as pulse oximeters or spirometers.

Gusmer [14] and Mochari-Greenberger [15] studied populations experiencing multiple medical and psychiatric diagnoses, chronic disease management conditions, treatment adherence, patient outcomes and compliance with prescribed treatment plans. In treating the whole person, mental health counselors may be called upon to work with medical and allied health professionals in providing effective patient care. More specifically, wearable remote monitoring for physical health conditions have included autoimmune disorders [16]; obesity [17,18]; psychiatric disorders [19], type 1 diabetes and glycemic control [20,21]; pulmonary rehabilitation [22]; asthma [23], cancer [24]; dementia and cognitive decline [12]; weight management and obesity [17]. There have been a number of studies through the Department of Veterans Affairs targeting specific populations including health informatics, home telehealth, and disease management in support of the care of veteran patients with chronic conditions [25]. More specifically, noninvasive technologies are now commonly being integrated into disease management strategies to provide additional patient information, with the goal of improving healthcare decision-making. Digital technologies are continually being adopted as an additional method for healthcare systems to increase patient contact and augment the practice of psychotherapeutic care and treatment.

SMART technologies can provide mental health counselors with the option of monitoring patients for compliance and sharing health data with remotely based clinical experts for consultation, saving time and expense for practitioners and patients, and actively managing treatments for those with chronic conditions. The accuracy, validity and reliability of SMART trackers has been reported as significantly beneficial in counseling care [26]. Health data are typically transmitted to healthcare professionals in facilities such as monitoring centers in primary care settings, hospitals and intensive care units, skilled nursing facilities, and centralized management programs, among others. Figure 1 provides a schematic for this model of healthcare delivery.

Figure 1 summarizes the core elements of SMART technology available for use with mental health interventions. Patient motivation is a critical element in therapeutic change and can best be measured by patient commitment and compliance with prescribed treatment interventions. It assumes an understanding of patient's readiness to change [27] and the importance of working with a motivated patient using SMART technology as an adjunct to counseling and psychotherapy strategies.

\section{Counseling Intervention Model}

A contemporary counseling approach that matches well with SMART technology use is a Dialectical Behavior Therapy [28] model and utilizing available SMART technology for monitoring compliance and behavior changes with a patient. Dialectical behavior therapy (DBT) combines both cognitive and behavioral techniques for treating individuals. Dialectical Behavior Therapy (DBT) is a cognitive behavioral treatment that was originally developed to treat chronically depressed and suicidal individuals 
diagnosed with borderline personality disorder (BPD). Research has demonstrated that it is effective in treating a wide range of other disorders such as anxiety disorders, substance dependence, multiple health conditions associated with anxiety and eating disorders. Using a modified DBT model including individual health counseling utilizing patient relevant micro skills [29], including the use of smartphone consultation, and health team consultation provides a conceptual basis for certain diseases and mental health disorders.

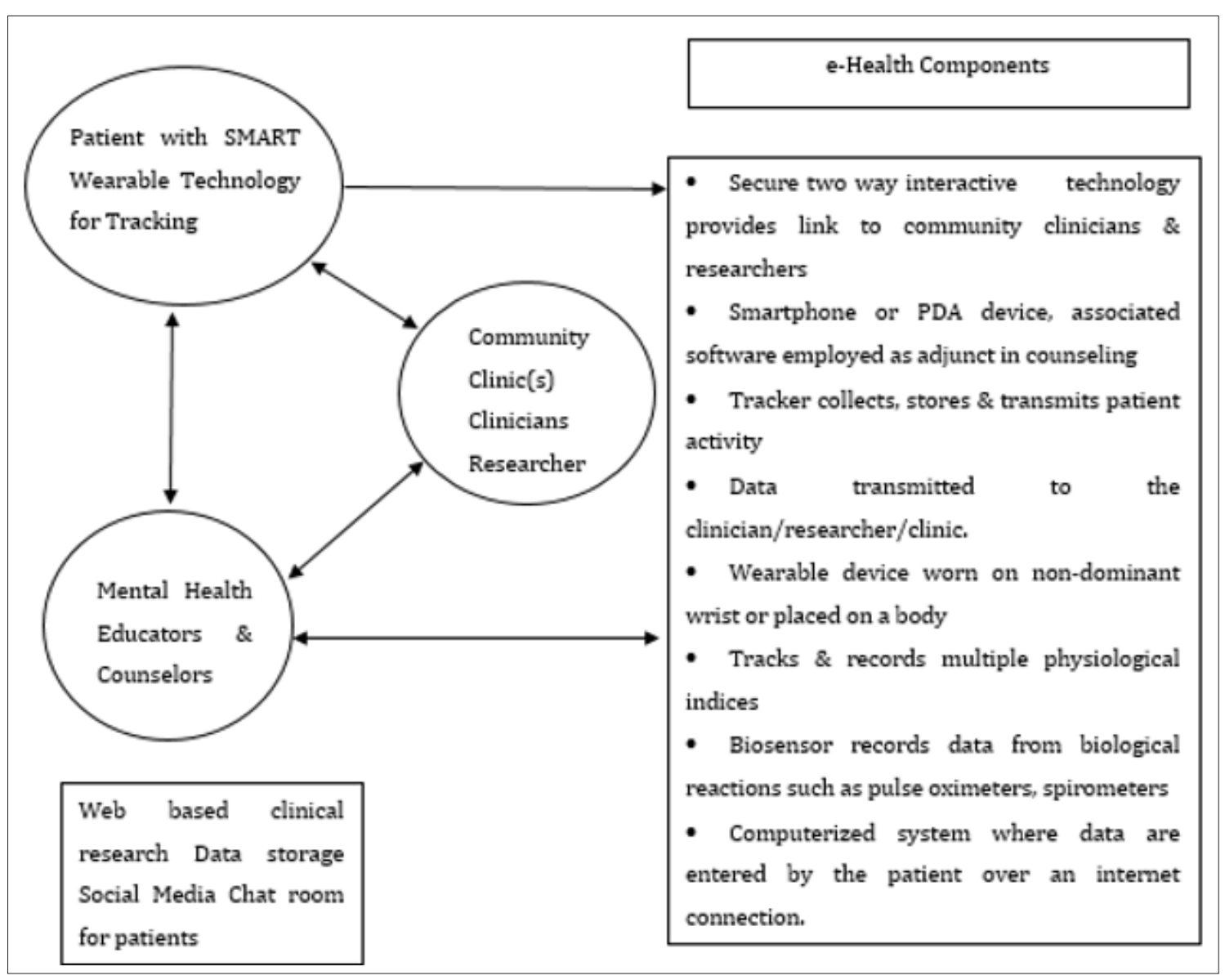

Figure 1: SMART Technology Model in Health Related Counseling.

\section{Case Study}

For the purpose of mental health counseling, the integrated outpatient clinical team referred a patient who presents with anxiety associated with elevated lipids, high blood pressure, and obesity is referred to the outpatient multidisciplinary team. The patient shows little understanding of the implications of his health and a sedentary life style. He is diagnosed with both a psychiatric and physical health set of diagnoses. These include a diagnosis of anxiety due to medical conditions that include type 2 diabetes with complications of obesity, hypertension and cardiovascular disease. He was judged to be at the contemplative level of stage of change [27]. Eight sessions over sixteen weeks involved short term, modified Dialectic Behavior Therapy (DBT) focusing on triggers, thoughts, behaviors that impair his ability to change and helping the patient understand the implications of doing nothing about their physical condition. Examining the patient options to better manage his health condition, the use of SMART wearable technology was introduced to the patient. Two sessions focused on a review of the benefits of its measurable use to monitor physical activity, along with sleep monitoring, exercise activity levels and calorie control for his diabetes, coronary artery disease and other medical problems.

The focus in counseling is on the patient's thoughts, feelings and bodily sensations related to his current health and medical conditions were explored along with managing his condition. This was crucial in creating a mindful recognition and commitment to assuming some responsibility for addressing it. From a dialectical behavior therapy standpoint, maladaptive behaviors continue because the consequences of the behavior provide some kind of reinforcement. For example, if the patient fails to assume a more active lifestyle, more closely managing his food and calorie intake and fails to monitor sleep habits and patterns, then one can only expect that more serious medical concerns will result and the anxiety associated with these more serious medical problems will find no relief. This pattern of behavior will lead to cardiovascular complications along with peripheral neuropathy which may result 
in serious limitations in his life. Recent research [30] has provided support for applying SMART technology for this patient with anxiety and multiple health related problems.

The next session focused on the use of a wearable device for remote monitoring using of a FitBit device synchronized to his Smartphone for tracking. The use of the Fitbit measures several variables related to health and wellness. The mechanism as to how this SMART technology works is best explained by understanding that it is a synchronized tracker that use a 3-axis accelerometer to understand one's physical movement to assess level of activity. By analyzing acceleration data, the tracker provides detailed operational information about frequency, duration, intensity, and patterns of movement to determine steps taken, distance traveled, calories burned, and sleep quality. Using a self-monitoring device to gauge changes over time that addressed physical activity, sleep patterns, weight and calorie intake and expenditure, He agreed through a behavioral contract to increase exercise on a graduated basis and agreed to work with a registered dietician to set goals that would restrict caloric intake to 1,500 calories per day, using a diet of nutritionally balanced supplements and one daily meal, and self-monitoring.

Issued to the patient was a Fitbit One which is a wireless activity tracker that syncs using Bluetooth SMART technology on the patient's personal Android smartphone. This technology permits the patient to record several daily activities including but not limited to the number of steps taken, distance walked or ran, on foot, calories burned by the patient during activity periods, sleep efficiency periods of movement during sleep, number of wake ups during sleep and other personal biomarkers.

The patient was trained to use, store, tabulate, self-enter certain data and recharge the Fitbit. Specificity in self-entered data included how to enter the logging of food consumption, activities, water intake and weight monitoring, as well as track fitness goals throughout the day even while offline. Tracking assessments were chosen to detect changes in management related activities. Fitbit is an American made product which is developed and designed to measure biomarkers that include several personal metrics. The Fitbit technology is worn on the non-dominant wrist and includes an activity tracker. This type of wireless-enabled wearable technology device measures and stores data that can be transmitted to clinical and research professionals when research participants elect to "share" their FitBit page and information with research professionals.

Measurable results revealed that the patient began to realize short term goals early on which served as a motivator to him for continued use. The initial benefit was a five-pound weight loss the first week. He continued to lose weight fairly steadily with a total weight reduction of 11 pounds after 16 weeks. In addition to the obvious benefits of enhanced self-esteem and healthier eating habits, this patient showed signs of enjoying some very important, perhaps life-saving, health benefits. His cholesterol was reduced. Prior to starting the Fitbit, his cholesterol was 265 and his triglyceride was 478 . More recently, his lipids were greatly improved, with a cholesterol of 156 and a triglyceride of 138 . His blood pressure was reduced to $118 / 84$ at the end of the 16 week period. His fasting blood glucose was reduced from 311 to 109. After the 16 week intervention program, results revealed significant qualitative improvements in the active minutes, steps taken and miles walked by the patient. In addition, the technology reported on calories burned, hours slept and water consumed during each 24 hour period had achieved normal levels.

By using the SMART technology, the counselor was able to monitor changes made over time and noted improved time dedicated to exercise, walking and total miles walked. On miles walked per day, the results show that the patient increased miles walked from less than one mile per day to more than 4.3 miles per day which approached the recommended 5 miles per day or 10,000 steps recommended by the Surgeon General (US Surgeon General 2012). The calorie monitor aided the patient in changing calorie intake from that which exceeded 3500 calories per day to the recommended 2500 calories per day. Furthermore, the sleep total hours slept per night changed from less than 5.8 per night to 8.5 , as encouraged by his physician.

\section{Discussion}

The wearable SMART device Fitbit shows potential for patients with current health related psychotherapeutic interventions who would benefit from accurately monitoring multiple behavioral activities including but not limited to physical activity, calorie intake and expenditure, and sleep patterns of patients. The case study patient appears to have benefited from a healthy diet and exercise program. Most people are surprised to find that they have vastly underestimated their caloric intake and overestimated their physical activity. Also, many people overestimate or underestimate their weight problem. Often, seriously overweight people spend much of their lives struggling to lose weight, jumping from one fad diet or new remedy to another without ever achieving lasting success. The problem isn't necessarily a lack of motivation; they may have simply been misguided by mixed messages in the media or friends and less than accurate caloric intake and caloric expenditure. Utilization of SMART technology along with an effective diet and exercise program professional guidance and practice among allied health professionals can make all the difference for these patients in achieving improved and recommended health goals including weight, diet, sleep, exercise, self-esteem and an improved quality of life.

The use of wearable technology devices like the popular Fitbit has become a popular health \& fitness gauge for about a decade. Health and wellness prone individuals' benefit from self-tracking using such a device as they provide a variety of self-monitoring information and biomarkers. When one begins to catch on to selfmonitoring various health and wellness factors, one begins to compete with oneself. It can drive almost anyone to check weight on a daily basis, exercise more, monitor sleep cycles and patterns, and help detect mood shifts over time.

Some of the current SMART trackers such as Lintelek Slim, Garmin Vivofit, Fitbit Alta, Quantified Self, Nike Fuel band, Jawbone 
provide mental health counselors, patients and researchers with accurate measurable data in tracking and understanding change during psychotherapeutic interventions. There are alarms that aid in monitoring when one exceeds caloric intake against the amount of calories burned through various forms of exercise during the day. For example, one can make sure that he/she hits a daily minimum calories burned that they have set for themselves. Knowing ones overall daily caloric intake and expenditure and how many calories one burns per day via exercise, seems to be very useful information to have in self-monitoring. The usefulness of such devices for behavioral management for weight will be a direct function of how much it helps each individual using it and how compliant they are with accurately getting calories expended greater than calories consumed over time.

Eventually the patient who has become accustomed to such technology can self-monitor after termination from treatment. Reasonable goal-setting is critical to engagement and compliance. The patient in this pilot study indicated this helped him and he was more likely to follow through on prescribed treatment. Receiving virtual rewards such as ribbons, stars or badges was not motivating nor that helpful. The accomplishment of set goals on a daily basis was most effective reward.

Fitness trackers permit patients to track progress in the form of short-term goals such as number of steps walked per day before they reach some longer, more difficult goals such as weight loss, which may be key to motivation. Wearable technologies can measure heart beats, count steps and monitor your sleep are bringing a new dimension to health and wellness, but the person is still responsible for maintaining healthy indicators, exercise and rest. There are limitations to be noted with the technology and with the design and methodology used in this pilot study. A case study design is often viewed as size and lack of group randomization were a limitation. Multiple subject case design would be beneficial and this case study can serve as the pilot for multiple subjects along with a comparison study using multiple models of the wearable technology beyond FitBit such as the Smartwatch, Jawbone, Microsoft Band, Quantified Self or Smart keychain. This effort to pilot the use of wearable technology as an adjunct to an evidence based model of psychotherapeutic intervention offers promise in both clinical and research assessing prevention interventions as a part of counseling.

Clinical data captured by these SMART technologies signal promise for application and accuracy of measurement include vital signs, weight, blood pressure, oxygen levels, and heart rate $[11,14]$. The future is likely to bring greater and more rapid technological advances, opportunities for academic health centers to expand their reach, and changes to the nature of medical care. In the near term, many advances will probably be linked to smartphones, which $90 \%$ of the world population will have in another decade. The increasingly sophisticated sensors and growing number of peripheral assessments may enable smartphones to monitor a person's health passively, facilitate diagnosis, and connect patients to clinicians when needed.
From a clinical research perspective, the ability to monitor patient-generated real data over time by using sensors, laboratories, and imaging, comes with security and privacy concerns. Telehealth applications can enable academic health centers and university training programs the opportunity to expand their reach by improving access for patients [3]. With the growing global burden of chronic conditions academic health centers can use telehealth to reach many clinicians and their patients globally. Such efforts could expand and even integrate many of the health services that are currently provided by academic health centers. The reputations of these academic health centers could be especially helpful for engendering trust in patients who may receive care from clinicians whom they have not actually met in person.

The growth of the use of SMART technology through telehealth applications over the last decade and future advances in this field will have serious implications for health care delivery and prevention efforts. The provision of care at a distance could help address longstanding concerns about the distribution and availability of health care providers [2]. The migration of care from hospitals and clinics to the home and smart-phones may also eventually decrease the demand for clinic space, a trend that is occurring today. The nature of a patient "visit" will also change because telehealth will not seek to replicate traditional office visits but rather capitalize on its unique strengths to define new care paradigms that improve health. For example, rather than periodic, highly structured inclinic encounters, visits may be shorter and more frequent and may occur on an as needed basis through SMART technology.

Recent research trends have used different terminology to capture the essence of e-Health and the overall language used to describe the benefits of wearable SMART technologies as an adjunct to health psychotherapy, there is clear evidence that the utility and benefits of such technology is being established and beneficial to mental health interventions [31-33]. SMART technology advances for improving healthcare delivery, wearable appliances that form an integral part of our personal life have emerged.

\section{Final Thoughts}

It is becoming increasingly clear that the use of 21st century technology is shaping the delivery of clinical care as well as the experience and training in counseling. On the positive side, technology's integration with mental health counseling is inevitable and show favorable potential to assist with the counseling process. Another benefit is that counselors assess with actual real life measures changes in behavior that promote prevention and hold the potential for improving health. Because of the advances in technology, contemporary counseling interventions are able to offer patient's strategies for helping them improve their lives. Wearable smart technology is one of those current advances that are providing an adjunct to the insight gained and cognitive behavioral advances realized in the provision of mental health counseling.

The use of SMART technology with mental health care raises a number of concerns. The mental health community, professional organizations and licensing boards along with hardware and 
software developers, must address standards to help consumers know if an app or other mobile technology is proven to be effective. The question of who will or should regulate mental health technology and the data it generates needs to be answered. Another concern is understanding if apps work for all clients and for all mental health conditions. The issue of confidentiality is so very important. Apps deal with very sensitive personal information so app makers need to be able to guarantee privacy for app users.

This single case study design has served as a pilot with the realization that there are a number of limitations. It lacks a multiple subject design model and randomization to assess the effectiveness of multiple models of the wearable technology. What it represents is an introduction to the use of SMART wearable technology in monitoring and promoting self-monitoring among patients who experience anxiety related to medical condition that could benefit from life style changes. Mental health counselors may find this model of health care counseling as beneficial and researchers may use this as a basis for comparative research models investigating the effectiveness of SMART technology as an adjunct to counseling interventions.

It is important for mental health counselors to understand the SMART technology and the web culture of clients and to practice in an ethically responsible manner in an age of new technological advances [32]. In doing so, we have examined the adjunct use of SMART technology effectiveness in self-monitoring behavior change with a patient motivated to utilize smart technology in complying with prescribed clinical treatment. An integrated clinical team approach involving a mental health counselor, physician, registered dietician and telecare specialist worked together in the treatment of a person with both physical and mental health needs.

An exploratory case study research design was employed to explore those situations in which the intervention being evaluated has no clear, single set of outcomes. It was hypothesized that using a wearable self-monitoring device while involved in the treatment of multiple medical conditions would be beneficial to behavioral compliance and behavioral change in a motivated patient being treated by a multidisciplinary integrated treatment model including allied health professionals. After the 36 week intervention program, results revealed significant qualitative improvements in the active minutes, steps taken and miles walked by the patient. In addition, the technology reported on calories burned, sleep hours and minutes logged and water consumed during each 24 hour period. The integrated allied health team was able to monitor changes made over time and noted improved multiple outcomes. Wearable devices are always on and accessible and their ability to help manage, sort, and filter information will become more intimately connected to our daily lives. Health promotion science will benefit from the use of such technology. Wearable devices can be beneficial to assisting mental health counselors in monitoring effective behavioral changes of patients over time.

\section{Acknowledgement}

The authors wish to acknowledge the assistance of Thomas Holcomb, Ed.D, Professor Emeritus and former Chair of the
Department of Educational Studies, Leadership and Counseling, Murray State University, Murray Kentucky and the supervising psychiatrist Robert F. Kraus M.D. for their contributions to the completion of this manuscript.

\section{References}

1. Myers Kathleen, Nelson Eve-Lynn, Rabinowitz Terry, Hilty Donald (2017) American Telemedicine Association Practice Guidelines for Telemental Health with Children and Adolescents Telemedicine and e-Health 23(10): 779-804.

2. Miller TW, Wood J (2012) Telepractice: A $21^{\text {st }}$ Century Model of Health Care Delivery. In: Guah, Matthew (Edn.); Healthcare Delivery: Reform and New Technologies. New York: Information Science Publishers, ISBN: 1609601831.

3. Bashshur RL, Howell JD, Krupinski KA, Harms KM, Bashshur N, et al. (2016) The Empirical Foundations of Telemedicine Interventions in Primary Care. Telemedicine \& e-health 22(5): 342-358.

4. American Counseling Association (2014) Code of Ethics, telehealth.

5. American Mental Health Counselors Association (2015) Policy on Telehealth. I.B.6.

6. National Board for Certified Counselors (2012) Policy regarding the Provision of Distance Professional Services.

7. DeLucia PR, Harold SA, Tang YY (2013) Innovation in technologyaided psychotherapy through human factors/ergonomics: Toward a collaborative approach. Journal of Contemporary Psychotherapy 43(4): 253-260.

8. Steinhubl S, Muse E, Topol E (2015) The emerging field of mobile health. Sci Transl Med 7 (283): 283rv3.

9. Wang JB (2015) A Wearable Sensor (Fitbit One) and Text-Messaging to Promote Physical Activity and Participants' Level of Engagement (A Randomized Controlled Feasibility Trial) (Doctoral dissertation, University of California, San Diego).

10. Chan M Jean-Yves Fourniols, M Christophe Escriba, C Eric Campo E (2012) Smart wearable systems: Current status and future challenges. Artificial Intelligence in Medicine 56(3): 137-156.

11. Field MJ, Grigsby J (2002) Telemedicine and remote patient monitoring. JAMA 288: 423-425.

12. Allard M, Husky M, Catheline G (2014) Mobile technologies in the early detection of cognitive decline. PLoS One 9(12): e112197.

13. American Telemedicine Association (2015) Telemedicine definition.

14. Gusmer RJ, Bosch TA, Watkins AN, Ostrem JD, Dengel DR (2014) Comparison of FitBit $\AA$ Ultra to Acti Graph ${ }^{\text {TM }}$ GT1M for Assessment of Physical Activity in Young Adults during Treadmill Walking. Open Sports Medicine Journal 8: 11-15.

15. Mochari-Greenberger H, Vue L, Luka A, Peters A, Pande RL (2016) A Tele-Behavioral Health Intervention to Reduce Depression, Anxiety, and Stress and Improve Diabetes Self-Management. Telemedicine \& e-Health 22(8): 624-630.

16. Hamine S, Gerth-Guyette E, Faulx D, Green BB, Ginsburg AS (2015) Impact of mHealth chronic disease management on treatment adherence and patient outcomes: A systematic review. J Med Internet Res 17(2): e52.

17. Allen JK, Stephens J, Dennison Himmelfarb CR, Stewart KJ, Hauck S (2013) Randomized controlled pilot study testing use of smartphone technology for obesity treatment. J Obesity 15: 1597-1599.

18. Nollen NL, Mayo MS, Carlson SE, Rapoff MA, Goggin KJ, et al. (2014) Mobiletechnology for obesity prevention: A randomized pilot study in racial- and ethnic-minority girls. Am J Prev Med 46(4): 404-408.

19. Faurholt-Jepsen M, Ritz C, Frost M (2015) Mood instability in bipolar disorder type I versus type II-continuous daily electronic self-monitoring of illness activity using smartphones. J Affect Disord 186: 342-349. 
20. Franc S, Borot S, Ronsin 0 (2014) Telemedicine and type 1 diabetes: Is Technology per se sufficient to improve glycaemic control? Diabetes Metab 40(1): 61-66.

21. Fukuoka Y, Gay CL, Joiner KL, Vittinghoff E (2015) A novel diabetes prevention intervention using a mobile App: A randomized controlled trial with overweight adults at risk. Am J Prev Med 49(2): 223-237.

22. DeVito Dabbs A, Dew MA, Myers B (2009) Evaluation of a hand-held, computer based intervention to promote early self-care behaviors after lung transplant. Clin Transplant 23(4): 537-545.

23. Ryan D, Price D, Musgrave SD (2012) Clinical and cost effectiveness of mobile phone supported self-monitoring of asthma: Multicentre randomised controlled trial. BMJ 344: e1756.

24. Kearney N, McCann L, Norrie J (2009) Evaluation of a mobile phonebased, advanced symptom management system (ASyMS) in the management of chemotherapy-related toxicity. Support Care Cancer 17(4): 437-444.

25. Darkins A, Ryan P, Kobb R, Foster L, Edmonson E, et al. (2008) Care Coordination/Home Telehealth: The systematic implementation of health informatics, home telehealth, and disease management to support the care of veteran patients with chronic conditions. Telemed J E Health 14(10): 1118.

26. Mammen G, Gardiner S, Senthinathan A, McClemont L, Stone M, et al. (2012) Is this Bit Fit? Measuring the Quality of the Fitbit Step-Counter The Health \& Fitness Journal of Canada 5(4): 30-39.

\section{ISSN: 2574-1241}

DOI: 10.26717/BJSTR.2018.08.001609

Thomas W Miller. Biomed J Sci \& Tech Res

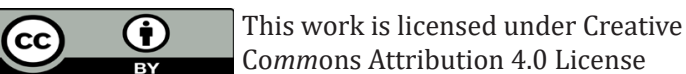

Submission Link: https://biomedres.us/submit-manuscript.php
27. Prochaska JO, Norcross JC (2013) Systems of psychotherapy: A transtheoretical analysis ( $8^{\text {th }}$ edn.); Pacific Grove, CA: Brooks/Cole.

28. Linehan M (2016) Behavior Therapy: Where We Were, Where We Are and Where We Need to Be Going Cognitive and Behavioral Practice. New York: Elsevier Ltd 13: 1077-7229.

29. Ivey A, Authier J (1978) Micro-counseling: Innovations in Interviewing, Counseling Psychotherapy \& Psychoeducation. Thomas Publishers.

30. Mochari-Greenberger H, Vue L, Luka A, Peters A, Pande RL (2016) A Tele-Behavioral Health Intervention to Reduce Depression, Anxiety, and Stress and Improve Diabetes Self-Management. Telemedicine \& e-Health 22(8): 624-630.

31. United States, Office of the Surgeon General (2012) Shape Up America. Washington DC: The U.S. Surgeon General's Guidelines on Promoting Physical Activity among All Americans.

32. Nagel DM, Anthony K (2009) Ethical Framework for the Use of Technology in Mental Health.

33. Munson SA, Consolvo S (2012) Exploring goal-setting, rewards, selfmonitoring, and sharing to motivate physical activity. In Pervasive Computing Technologies for Healthcare (Pervasive Health), $6^{\text {th }}$ International Conference on IEEE, pp. 25-32.

AlOMEDICAL
RESEARCHES $\quad \begin{aligned} & \text { Assets of Publishing with us } \\ & \text { - Immediate, unrestricted online access }\end{aligned}$

